\title{
On background-independent renormalization in spin foam models
}

\author{
Benjamin Bahr* \\ II. Institute for theoretical physics \\ University of Hamburg \\ Luruper Chaussee 149 \\ 22761 Hamburg \\ Germany E-mail: benjamin.bahrddesy.de
}

In this talk a brief interpretation of renormalization group flow in a background-independent context will be presented. It will be applied to spin foam models, which provide tentative examples of quantum gravity path integrals. The notion of continuum limit is intimately interwoven with this notion of background-independent RG flow. Diffeomorphism-invariance in this context will be discussed, and examples of models with and without that invariance will be presented.

Frontiers of Fundamental Physics 14 - FFP14,

15-18 July 2014

Aix Marseille University (AMU) Saint-Charles Campus, Marseille

\footnotetext{
* Speaker.
} 


\section{Motivation}

The Wilsonian idea of renormalization group flow is a quite useful tool for describing physical theories with a large number of degrees of freedom, most notably quantum field theories or statistical systems [ [ ㅁ] ]. At its core lies the notion of scale: the degrees of freedom are not all considered simultaneously, but ordered along a hierarchy, often given by a length (or energy-) parameter. The effective dynamics for the degrees of freedom at a certain scale are given by an action with scale-dependent coupling constants.

Because most physical theories are non-linear, i.e. exhibit non-trivial self-interaction, and because large scale degrees of freedom are more often than not collective phenomena of small scale degrees of freedom, the coupling constants on different scales are not independent of each other. Rather, the renormalization group flow describes how the physics on small (UV) scales determines the physics at large (IR) scales.

It has always been difficult to apply these ideas to (quantum) general relativity, due to its lack of background structure. Unlike most other physical theories, the group of space-time diffeomorphisms is a gauge symmetry of the theory. Hence, there are no fixed structures which are not invariant under diffeomorphisms, in particular no metric with respect to which one could measure length- or energy scales. Rather, in general relativity the metric itself is one of the fields, which a priori prevents the ordering of field excitations along a given length scale.

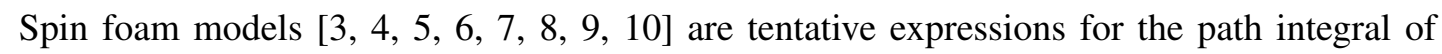
quantum gravity in terms of a discrete state sum. One of the most used models is the EPRL model [四], which prescribes a specific set of discrete data - corresponding to a discrete geometry - and a certain amplitude function, which contains the dynamics of the model. Essentially, it can be viewed as a generalized version of lattice gauge theory on an irregular lattice. Notably, the lattice edges do not come with a prescribed length. Rather, the discrete data on the lattice can be used to extract geometric information of the lattice.

In this talk we will describe how to introduce a notion of scale to spin foam models, providing a hierarchy of degrees of freedom. This will result in a notion of renormalization group flow, and we will show how it can be used to construct rigorous path integral measures of the theory.

It should be noted that this notion of RG flow is independent of the physical interpretation of the used discretization. Currently there are two major interpretations: Firstly, the discretization can be seen as a cutoff of the degrees of freedom. The amplitudes associated to a cut-off are therefore effective actions, describing the effective dynamics of the degrees of freedom which are associated to the discrete lattice (a point of view adopted e.g. in [ए]]). On the other hand, one can interpret the lattice in itself as fundamental, providing a notion of discrete space-time, together with an action describing the dynamics fundamental degrees of freedom.

Independent of which interpretation is adopted, the RG flow described in this article is a crucial part of the analysis of the resulting theory, albeit that the outcome may have different physical interpretations attached to it. In the first case, the RG flow describes the change of effective dynamics associated to different scales, each given by a specific choice of discretization. In this case, one would have to solve the RG flow equations and try to find those (generalized) RG trajectories which lead to the correct large-scale behaviour, i.e. general relativity. In the second case, there might be a fundamental lattice, corresponding to the Planck scale, with a specified model on it (such as 
the EPRL model). In that case one would have to solve the RG flow equations in order to check whether the long-range degrees of freedoms behave accordingly to general relativity, i.e. whether the quantum theory has the correct IR limit.

\section{Basic definitions}

In what follows, we briefly describe a general class of models we will use as setup. These are slightly generalized versions of the holonomy spin foam models described in [127, [13, [4]]. Most of contemporary spin foam models, as well as lattice gauge theory, can be brought into this form.

We model space-time by a semi-analytic manifold $M$. The local gauge group will be given by a compact Lie group $G$. Consider the collection of all finite, regular, semi-analytic 2-complexes $\Gamma$, embedded in $M .{ }^{1}$ For each $\Gamma$, denote its vertices, edges and faces by $v=1, \ldots V, e=1, \ldots E$, and $f=1 \ldots F$, respectively. Each edge and face is assigned an orientation.

For a 2-complex $\Gamma$ define $E \ltimes F$ to be the set of all pairs $(e, f)$ such that $e$ is in the boundary of $f$. Then the configuration space for $\Gamma$ is given by

$$
\mathscr{A}_{\Gamma}:=G^{E \ltimes F},
$$

i.e. the set of all distributions of group elements in $G$ to pairs $(e, f)$ with $e \subset \partial f$.

Predictions of physical theories are given in terms of expectation values of observables. For a 2-complex $\Gamma$, the space of observables is given by continuous functions on $\mathscr{A}_{\Gamma}$, i.e. elements in $C^{0}\left(\mathscr{A}_{\Gamma}\right)$. Expectation values are hence given by (positive, normalized) linear functionals on $C^{0}\left(\mathscr{A}_{\Gamma}\right)$, which by the Riesz representation theorem correspond to regular (normalized) Borel measures $\mu_{\Gamma}$ on $\mathscr{A}_{\Gamma}$. The expectation value of an observable $\mathscr{O}_{\Gamma}$ is therefore given by

$$
\left\langle\mathscr{O}_{\Gamma}\right\rangle_{\Gamma}=\int_{\mathscr{A}_{\Gamma}} d \mu_{\Gamma}\left(\left\{h_{(e, f)}\right\}\right) \mathscr{O}_{\Gamma}\left(\left\{h_{(e, f)}\right\}\right)
$$

The measure $\mu_{\Gamma}$ should be interpreted as the effective path integral measure at the scale given by the 2-complex $\Gamma$. The measure includes all dynamical information, in particular about the (effective) action. For example, on a regular lattice $\Gamma_{a, \text { reg }}$ of lattice length $a$, the measure for lattice gauge theory is given by

$$
d \mu_{a}\left(\left\{h_{(e, f)}\right\}\right)=\frac{1}{N} \int_{G^{E}} d \mu_{\mathrm{Haar}}\left(\left\{g_{e}\right\}\right)\left(\prod_{e} \prod_{f \supset e} \delta\left(g_{e}, h_{(e, f)}\right)\right) \exp \left(-S^{(a)}\left(\left\{g_{e}\right\}\right)\right)
$$

where $S^{(a)}$ is the effective action at scale $a$, containing, for example, the Wilson action.

Note how the measure in $(\sqrt{2.3})$ contains a product of Dirac delta functions, which force all $h_{(e, f)}$ for one $e$ to be equal to some $g_{e}$. In other words, the support of the measure is on some set $G^{E} \subset G^{E \ltimes F}$. This is because the link variables $g_{e}$ are the important physical quantities in gauge theory. The spin foam models we will be concerned with are slightly more general, in that they allow the $h_{(e, f)}$ to fluctuate independently, as a result of imposing the simplicity constraints. See also [1]4]

\footnotetext{
${ }^{1}$ See [피] for details on the definitions.
} 


\section{Renormalizing and continuum limit}

Each of the 2-complexes $\Gamma$ plays the role of a scale, in the sense that it defines the degrees of freedom $h_{(e, f)}$ which can be measured at the scale $\Gamma$. Since each group element $h_{(e, f)}$ can be seen as the integration of a continuum connection along some path [뜨, 때, 띠], one needs all $h_{(e, f)}$ for all possible $\Gamma$ embedded in $M$, to get back the information about the continuum degrees of freedom. Moreover, the $h_{(e, f)}$ on different $\Gamma$ are not independent of each other, because different $\Gamma$ 's are not.

The continuum limit is precisely given by an assignment of a measure $\mu_{\Gamma}$ to each $\Gamma$ embedded in $M$, which is consistent in the sense that it respects these interdependencies. This is what we will formalize in what follows.

The set of 2-complexes possesses a partial order $\leq$ : we say that $\Gamma \leq \Gamma^{\prime}$ if each $n$-cell of $\Gamma$ is a union of $n$-cells of $\Gamma^{\prime}$, for $n=0,1,2$. Then $\leq$ obeys transitivity, as well as the following important property: For any two 2-complexes $\Gamma_{1}$ and $\Gamma_{2}$, there exists a third 2-complex $\Gamma$ such that both $\Gamma_{1} \leq \Gamma$ and $\Gamma_{2} \leq \Gamma$ hold (here the semi-analyticity of $M$ and of the $\Gamma$ 's come into play).

For $\Gamma \leq \Gamma^{\prime}$ we define the surjective homeomorphism

$$
\pi_{\Gamma^{\prime} \Gamma}: \mathscr{A}_{\Gamma^{\prime}} \longrightarrow \mathscr{A}_{\Gamma}
$$

given by

$$
\left(\pi_{\Gamma^{\prime} \Gamma}\left(\left\{h_{\left(e^{\prime}, f^{\prime}\right)}\right\}_{\left(e^{\prime}, f^{\prime}\right)}\right)\right)_{(e, f)}:=\vec{\prod}_{e^{\prime} \subset e, f^{\prime} \subset f^{\prime}} h_{\left(e^{\prime}, f^{\prime}\right)}^{\left[e^{\prime}, e\right]},
$$

where the rhs of (3.2) denotes the ordered product of group elements along an edge $e$, and $\left[e^{\prime} e\right]= \pm 1$ is the relative orientation of $e^{\prime}$ and $e$.

Note that the $\pi_{\Gamma^{\prime} \Gamma}$ relate degrees of freedom on $\Gamma$ and $\Gamma^{\prime} \geq \Gamma$. In particular, they define a coarse graining of degrees of freedom in $\mathscr{A}_{\Gamma^{\prime}}$ to those in $\mathscr{A}_{\Gamma}$.

For $\Gamma \leq \Gamma^{\prime} \leq \Gamma^{\prime \prime}$ the coarse graining maps $\pi_{\Gamma^{\prime} \Gamma}$ obviously satisfy

$$
\pi_{\Gamma^{\prime} \Gamma} \pi_{\Gamma^{\prime \prime} \Gamma^{\prime}}=\pi_{\Gamma^{\prime \prime} \Gamma}
$$

A collection of measures $\left\{\mu_{\Gamma}\right\}_{\Gamma}$ is called cylindrically consistent (or promeasure), if for each $\Gamma \leq \Gamma^{\prime}$ one has that

$$
\left(\pi_{\Gamma^{\prime} \Gamma}\right)_{*} \mu_{\Gamma^{\prime}}=\mu_{\Gamma}
$$

This condition can be reformulated: consider an observable $\mathscr{O}_{\Gamma}$ associated to the scale $\Gamma$, then the pull-back to $\Gamma^{\prime}$ is given by $\mathscr{O}_{\Gamma^{\prime}}:=\mathscr{O}_{\Gamma} \pi_{\Gamma^{\prime} \Gamma}$. The interpretation is that of measuring the observable with a higher resolution, i.e. with more degrees of freedom. Since the observable only depended on the coarse grained degrees of freedom from the beginning, this should not give more information. In particular, the expectation values should coincide, i.e. $\left\langle\mathscr{O}_{\Gamma}\right\rangle_{\Gamma}=\left\langle\mathscr{O}_{\Gamma^{\prime}}\right\rangle_{\Gamma^{\prime}}$. This holding for all $\mathscr{O}_{\Gamma}$ is equivalent to (B.4). In other words, the measures being cylindrical consistency is equivalent to the different measures $\mu_{\Gamma}$ at different scales being compatible with each other.

In [15] it is shown how (B.4) also allows for the construction of a continuum measure $\mu$ on a space of (generalized) continuum fields $\overline{\mathscr{A}}$. So the continuum limit is not given in terms of an analytical limit of sorts, but as an effective action for each scale, satisfying (B..4) [ए]]. In the lattice 
gauge theory example, one can see that (B.4) reduces precisely to the Wilsonian RG flow equations, relating actions $S^{(a)}$ at different length scales, e.g. $a=2 a^{\prime}$.

Another comment is in order: In the case of a theory on a prescribed background metric, one can restrict oneself to, say, regular hypercubic lattices with certain edge lengths. Without such a background however, one is led to using all kinds of irregular lattices, given by the 2-complexes $\Gamma$. These do not possess a total order, only a partial order. The RG trajectory in the backgroundindependent case therefore does not run along a single parameter (such as a length- or energy scale), but along the whole partially ordered set given by all $\Gamma$ 's. The hierarchy along which the degrees of freedom are ordered is simply more complicated on the background-independent context. Still, the fundamental notions of Wilsonian RG flow can still be applied to this generalized situation.

\section{Diffeomorphism invariance}

In the spin foam approach to quantum gravity, the tension between the discrete structure of the 2-complexes and continuum diffeomorphism-invariance is pervasive [20, 201, 222, 223, 224, 201, 206, 227].

Because of the existence of the continuum limit of partial measures $\left\{\mu_{\Gamma}\right\}_{\Gamma}$, it makes sense to ask whether the continuum measure $\mu$ is diffeomorphism-independent. This is proliferated by the canonical action of the diffeomorphism group $\operatorname{Diff}(M)$ on the space of generalized continuum fields $\overline{\mathscr{A}}$. It should be clear that the condition

$$
\phi_{*} \mu=\mu, \quad \text { for all } \phi \in \operatorname{Diff}(M)
$$

is a strong condition for the partial measures $\mu_{\Gamma}$. In particular, if the $\mu_{\Gamma}$ are cylindrically consistent and the resulting continuum measure satisfies (4.]), the following necessarily holds:

For each 2-complex $\Gamma$, if there are two observables $\mathscr{O}_{1,2}$ on $\mathscr{A}_{\Gamma}$ such that there are 2-complexes $\Gamma_{1,2} \leq \Gamma$ and observables $\tilde{\mathscr{O}}_{1,2}$ on $\mathscr{A}_{\Gamma_{1,2}}$ with $\mathscr{O}_{i}=\tilde{\mathscr{O}}_{i} \pi_{\Gamma_{\Gamma}}$ for $i=1,2$, and there is a diffeomorphism $\phi \in \operatorname{Diff}(M)$ such that $\phi\left(\Gamma_{1}\right)=\Gamma_{2}$, then $\left\langle\mathscr{O}_{1}\right\rangle_{\Gamma}=\left\langle\mathscr{O}_{2}\right\rangle_{\Gamma}$.

This is a quite strong restriction on $\mu_{\Gamma}$, as one can readily see. Incidentally, the converse is also true: If $\mu$ is a measure on $\overline{\mathscr{A}}$ such that all partial measures $\mu_{\Gamma}$ satisfy the above condition, then $\phi_{*} \mu=\mu$ for all diffeomorphisms $\phi$. This way one can characterize diffeomorphism-invariant measures, by checking properties of partial measures.

\section{Examples}

There is an easy example, in which the main features of this RG flow picture can be discussed. A similar version has been treated in [ए8, [प]].

In this example, $M$ is a $2 \mathrm{~d}$ closed manifold, and we consider as $\Gamma$ all those 2-complexes which are a full singular decomposition of $M^{2}$. For such a $\Gamma$, and $G=U(1)$, we take an action of the form ([2.3) with $g_{e}=e^{i \phi_{e}}$ and

$$
S^{(\vec{a}, \vec{\theta})}\left(\left\{\phi_{e}\right\}\right)=\sum_{f}\left(\sum_{n_{f}}\left(\sum_{e \subset \partial f}[e, f] \phi_{e}+\pi n_{f}\right)^{2} / a_{f}+i\left(\theta_{f}+\sum_{e \subset \partial f}[e, f] \phi_{e}+\pi n_{f}\right)\right)
$$

\footnotetext{
${ }^{2}$ All remaining 2-complexes are coarser than one of those, so the respective partial measures can be deduced by cylindrical consistency.
} 
where the $a_{f}>0$ and $\theta_{f} \in \mathbb{R}$ are parameters depending on the faces $f$ in $\Gamma$. The constant $N$ in ([2.3]) is taken to be $\prod_{f}\left(2 \pi / a_{f}\right)^{-\frac{1}{2}}$ so that $d \mu_{\Gamma}^{(\vec{a}, \vec{\theta})}$ is normalized.

For $\Gamma \leq \Gamma^{\prime}$ and faces $f$ in $\Gamma$, the cylindrical consistency conditions (13.4) can easily be checked to be

$$
\sum_{f^{\prime} \subset f} a_{f^{\prime}}=a_{f}, \quad \sum_{f^{\prime} \subset f}\left[f^{\prime}, f\right] \theta_{f^{\prime}}=\theta_{f}
$$

where $\left[f^{\prime}, f\right]= \pm 1$, depending on whether the respective orientations of $f^{\prime}, f$ agree or disagree.

There are infinitely many obvious solutions to (5.2). For example, let $g$ be a metric, and $\theta$ a 2-form on $M$. Then $a_{f}:=\operatorname{Area}_{g}(f)$ and $\theta_{f}:=\int_{f} \theta$ obviously satisfy (5.2). The respective continuum measures $\mu^{g, \theta}$ on $\overline{\mathscr{A}}$ satisfy $\phi_{*} \mu^{g, \theta}=\mu^{\phi^{*} g, \phi^{*} \theta}$ for a diffeomorphism $\phi$, so no measure is diffeomorphism-invariant. It should be noted, however, that the (pointwise) limit $g \rightarrow 0$ exists as measure $\mu^{0, \theta}$ on $\overline{\mathscr{A}}$. So does the scaling limit $g \rightarrow \lambda g, \lambda \rightarrow \infty$, as measure $\mu^{\infty, \theta}$. The former ones contain the special case $\mu^{0,0}$, which is diffeomorphism-invariant, and is connected to the $U(1)$-version of the Dijkgraaf-Witten-invariant [ㄹ]. The second types of measures are all equal, independently of $\theta$, to the $U(1)$-version of the Ashtekar-Lewandowski measure on $M$. This is also diffeomorphism-invariant.

\section{Summary}

We have presented a framework for a background-independent renormalization scheme for spin foam models. The RG flow equations in this context are equivalent to the cylindrical consistency conditions of partial measures, which ensures the existence of a continuum measure.

Generically, the absence of a background structure leads to an uncountable set of equations (B.4). Also, the flow is not defined along a parameter, but along a partially ordered set, in our case the set of all embedded 2-complexes. It is with the introduction of a background metric that the usual notion of RG flow reappears: instead of having to use all 2-complexes, one can restrict to e.g. regular, equilateral lattices, which are just labelled by a length parameter.

The RG flow equations themselves are manifestly diffeomorphism-invariant, but their solutions generically spontaneously break diffeomorphism-invariance, introducing a background. Only very few solutions are actually diffeomorphism-invariant continuum measures. This shows that the notion of RG flow is specifically tied to the existence of a continuum limit. It is by no means guaranteed that this alone already guarantees the invariance of the resulting continuum limit under space-time diffeomorphisms. This has repercussions for quantum gravity, in which this invariance is a crucial part of the classical system. On the other hand, demanding the invariance of the measures under diffeomorphisms can already be imposed as conditions on the partial measures, and hence has the potential of severely restricting the set of allowed solutions. This could help with the search for interesting, four-dimensional, diffeomorphism-invariant continuum measures for quantum gravity.

\section{References}

[1] K. G. Wilson, Phys. Rev. B 4 (1971) 3174. 
[2] K. G. Wilson and J. B. Kogut, Phys. Rept. 12 (1974) 75.

[3] J. W. Barrett and L. Crane, J. Math. Phys. 39 (1998) 3296 [gr-qc/9709028].

[4] J. Engle, E. Livine, R. Pereira and C. Rovelli, Nucl. Phys. B 799 (2008) 136 [arXiv:0711.0146 [gr-qc]].

[5] L. Freidel and K. Krasnov, Class. Quant. Grav. 25 (2008) 125018 [arXiv:0708.1595 [gr-qc]].

[6] J. Engle, M. Han and T. Thiemann, Class. Quant. Grav. 27 (2010) 245014 [arXiv:0911.3433 [gr-qc]].

[7] A. Baratin and D. Oriti, Phys. Rev. D 85 (2012) 044003 [arXiv:1111.5842 [hep-th]].

[8] E. R. Livine and S. Speziale, Phys. Rev. D 76 (2007) 084028 [arXiv:0705.0674 [gr-qc]].

[9] L. Freidel and S. Speziale, Phys. Rev. D 82 (2010) 084040 [arXiv:1001.2748 [gr-qc]].

[10] A. Perez, Living Rev. Rel. 16 (2013) 3 [arXiv:1205.2019 [gr-qc]].

[11] E. Bianchi, C. Rovelli and F. Vidotto, Phys. Rev. D 82 (2010) 084035 [arXiv:1003.3483 [gr-qc]].

[12] W. Kaminski, M. Kisielowski and J. Lewandowski, Class. Quant. Grav. 27 (2010) 095006 [Erratum-ibid. 29 (2012) 049502] [arXiv:0909.0939 [gr-qc]].

[13] B. Bahr, F. Hellmann, W. Kaminski, M. Kisielowski and J. Lewandowski, Class. Quant. Grav. 28 (2011) 105003 [arXiv:1010.4787 [gr-qc]].

[14] B. Bahr, B. Dittrich, F. Hellmann and W. Kaminski, Phys. Rev. D 87 (2013) 044048 [arXiv:1208.3388 [gr-qc]].

[15] B. Bahr, arXiv:1407.7746 [gr-qc].

[16] B. Bahr, J. Phys. Conf. Ser. 360 (2012) 012042 [arXiv:1112.3567 [gr-qc]].

[17] B. Dittrich, arXiv:1409.1450 [gr-qc].

[18] R. Oeckl, Nucl. Phys. B 657 (2003) 107 [gr-qc/0212047].

[19] R. Oeckl, In *Rio de Janeiro 2003, Recent developments in theoretical and experimental general relativity, gravitation, and relativistic field theories, pt. C* 2296-2300 [gr-qc/0401087].

[20] B. Dittrich, arXiv:0810.3594 [gr-qc].

[21] B. Bahr and B. Dittrich, Class. Quant. Grav. 26 (2009) 225011 [arXiv:0905.1670 [gr-qc]].

[22] B. Bahr, R. Gambini and J. Pullin, SIGMA 8 (2012) 002 [arXiv:1111.1879 [gr-qc]].

[23] B. Bahr and B. Dittrich, Phys. Rev. D 80 (2009) 124030 [arXiv:0907.4323 [gr-qc]].

[24] B. Bahr, B. Dittrich and S. Steinhaus, Phys. Rev. D 83 (2011) 105026 [arXiv:1101.4775 [gr-qc]].

[25] C. Rovelli, arXiv:1107.2310 [hep-lat].

[26] B. Dittrich and S. Steinhaus, Phys. Rev. D 85 (2012) 044032 [arXiv:1110.6866 [gr-qc]].

[27] B. Dittrich, PoS QGQGS 2011 (2011) 012 [arXiv:1201.3840 [gr-qc]].

[28] R. Dijkgraaf and E. Witten, Commun. Math. Phys. 129 (1990) 393. 